\title{
A Cross-sectional Study of Cancer Knowledge and Beliefs Among Faith-based Chinese in the USA Versus Taiwan
}

\author{
Su-I Hou, Xian Cao \\ Health Management and Informatics, University of Central Florida College of Health and Public Affairs, Orlando, FL, USA
}

Objectives: This study examined cancer knowledge, beliefs, and practice among faith-based Chinese in the USA versus Taiwan to gain better understandings on how environment and culture might play a role for tailored cancer education programs.

Methods: A self-administered survey included a validated 10-item Cancer Screening Belief Scale (CSBS), an 8-item Cancer Screening Knowledge Test (CSKT), and a 14-item cancer Warning Signs Test (CWST) was administered. Participants were recruited from 9 Chinese churches (5 in the USA and 4 in Taiwan).

Results: A total of 372 Chinese participated, 50\% lived in the USA and 50\% in Taiwan. Mean age was 44.31 (standard deviation, 14.74), $60 \%$ males, and majority had college education (85\%). Taiwan participants scored higher on both CSKT (6.13 vs. 5.52; $p<0.001)$ and CWST ( 6.80 vs. $5.38 ; p<0.001)$. Although perceived screening benefits and barriers were similar, Taiwan participants endorsed higher on screening norms (11.67 vs. 10.82; $p<0.001)$. Taiwan participants also indicated more doctor recommending cancer screenings (42.1\% vs. $29.6 \%$; $p=0.015)$, USA participants were more likely to have had annual health exams (65.4\% vs. $48.9 \% ; p=0.002)$. Regression results showed that those resided in the USA were 2.38 times more likely to report annual health exams. Married status (odds ratio $[\mathrm{OR}], 2.85)$, college education $(\mathrm{OR}, 2.38)$, doctor's recommendation $(\mathrm{OR}, 2.87)$, no family cancer history $(\mathrm{OR}, 2.47)$, and those with lower barriers were significant factors on annual health exams.

Conclusions: Taiwan participants scored higher on cancer knowledge and screening norms, while more USA participants reported annual health exams. Taiwan's universal healthcare might play a role on the different healthcare seeking patterns.

Key words: Cancer screening, Faith-based, Knowledge, Beliefs, Taiwan, United States

\section{INTRODUCTION}

Cancer has been the leading cause of death among Chinese, with increasing rates since the 1980's [1]. In the USA, Asian

Received: April 6, 2017 Accepted: December 4, 2018

Corresponding author: Su-I Hou, DrPH

Health Management and Informatics, University of Central Florida College of Health and Public Affairs, 12805 Pegasus Drive, Orlando, FL 32816-1600, USA

E-mail: su-i.hou@ucf.edu

This is an Open Access article distributed under the terms of the Creative Commons Attribution Non-Commercial License (http://creativecommons.org/licenses/by$\mathrm{nc} / 4.0 / /$ which permits unrestricted non-commercial use, distribution, and reproduction in any medium, provided the original work is properly cited.
Americans is the only racial/ethnic group still experiencing cancer as the leading cause of death. The increasing cancer burden in Chinese populations could be tied to the rapidly growth and aging populations, as well as the westernized dietary, reproductive, and related lifestyles [2].

Asian Americans are among the largest immigrants and the fastest growing racial/ethnic group in the USA, representing $6.3 \%$ of the total USA population [3]. Chinese is the largest Asian subpopulation (23.0\%), with $69.0 \%$ born overseas [4]. However, current research show Chinese population is underrepresented in cancer research and reported poor adherence to screening guidelines. With barriers such as language fluency and cultural difference, Chinese immigrants face increased 
disparities in cancer screening participations $[3,5,6]$.

Recent trends of cancer incidence in Taiwan show a sustained and significant increase of $1.7 \%$ per year from 2002 to 2012, rates higher than other countries [1]. The Department of Health in Taiwan has actively implemented both primary and secondary prevention of major cancers, such as high-risk factor avoidance and periodical cancer screening, to reduce cancer incidence and mortality rates [7].

Chinese older adults living in the USA and Taiwan have different healthcare environment and language barriers. The National Health Insurance (NHI) program was launched in Taiwan in 1995 to provide health care for all residents [7]. Almost all (99\%) Taiwan residents participate in the program, which provides health care of acceptable quality, comprehensive benefits and convenient access to treatment. It accelerated health improvement in Taiwan [7]. On the other hand, language barriers and the complex USA healthcare system hinder healthcare access among Chinese immigrants in the USA $[5,6]$.

Lessons learned from the few existing studies examining cancer-screening among community and worksite groups in Taiwan reveal urgent need of preventive health services utilization [8-11]. Previous studies showed that only $62 \%$ of married and $31 \%$ of single women from a community sample reported a Pap test in the preceding 3 years [9], and less than $30 \%$ of a worksite sample had ever had a fecal occult blood test (FOBT) for colorectal cancers screenings [10]. Culturally tailored and innovative screening interventions in Taiwan have demonstrated effective to significantly encourage more nonadherent women in the intervention group than control group to receive a Pap test within 6 months (50\% vs. 32\%) [11], and encouraged $74 \%$ of a midlife workplace sample returned a completed FOBT within 4 weeks $[8,10]$.

Studies have shown that community-based recruitment strategies such as church or faith-based, are successful to reach diverse groups, especially the hard-to-reach and underserved individuals (e.g., recent immigrants and uninsured) $[12,13]$.

Existing faith-based cancer interventions mostly target African Americans [12] or Latino Americans [13]. Chinese church in the USA has been an important place to help immigrants understand western versus eastern perspectives and address language barriers among Chinese immigrants. Yet, little research has been done among faith-based Chinese American immigrants in the USA or those living in their native countries about their knowledge and beliefs towards cancer education.
The current study starts the initial attempt to address some of these important gaps on cancer education research among Chinese midlife and older adult populations. Specifically, this study examined cancer knowledge, beliefs, and practice among faith-based Chinese older adults living in the USA versus Taiwan to gain better understanding on how healthcare environment and culture might play a role for tailored cancer education intervention development.

\section{METHODS}

\section{Sample}

The current study is a cross-sectional study assessing cancer education related knowledge, beliefs, and practices among church-going Chinese adults. Five major Chinese churches in a large metropolitan city in the southeastern USA, and four Chinese churches in Taiwan participated in the study. The study samples were convenience samples and were selected based on community connections the research team had access to.

\section{Measurement}

Hou's 8-item validated Cancer Screening Knowledge Test (CSKT) $[10,14,15]$ and a 14-item Cancer Warning Signs Test (CWST) [14] were used to assess objective cancer knowledge in the current study. In addition, Hou's previously validated Cancer Screening Belief Scale (CSBS) [16] were used to measure theory-based towards cancer screenings in general. One subjective cancer knowledge item using 5-point Liker scale was used to measure participants' subjective cancer knowledge level [14,15]. Questionnaires were developed in English, translated into Chinese, and back-translated to ensure consistencies and accuracy of item meanings [8-11]. Both English and Chinese questionnaires were available for participants. The study was approved by the Institutional Review Board of University of Georgia, Athens, GA, USA (\#10789-2).

Hou's CSKT was originally developed to measure cancer knowledge related to cervical cancer screening $[9,11]$, and later expanded to measure knowledge related to cancer and screening of all cancer type $[14,15]$. This validated general cancer screening knowledge test included a total of 8 items on key cancer risk factors (age and family history), need for regular screenings, recommended guidelines of major ageappropriate cancer screenings (cervical, breast, colorectal cancers), and early stage signs. The CSKT instruments have been developed and tested among multiple Chinese middle and 
older-age adult samples in Taiwan, and consisted of a good mix of easy and moderate difficult items $[6,9,14,15]$.

The CWST measured the 7 cancer warning signs (C.A.U.T.I. O.N.) endorsed by the World Health Organization [17], and has been viewed as "The Ten Commandments" for cancer education. To thrive for a more balanced measurement scale, 7 noncancer warning signs were also added and tested [14]. Data from the current study showed that the reliability of the CWST $(n=14)$ was satisfactory (Cronbach alpha, 0.909$)$, with corrected-item-total-correlations (CITC) ranged 0.537 to 0.680 .

\section{Subjective cancer knowledge (1 item)}

Subjective knowledge was measured by one overall 5-Likert scale item on overall self-perceived (subjective) cancer knowledge level $[14,15]$. The item asked, "How would you rate your knowledge related to cancer and screenings in general?" This item was coded " 1 " as "very low" and " 5 " as "very high".

\section{Cancer screening belief scale}

Hou's previously validated CSBS [16] were used to measure theory-based beliefs towards cancer screenings in general. There were three sub-scales in the CSBS (total 10 items), perceived screening benefits (CSBS-Pros-3); perceived screening barriers (CSBS-Cons-4); and perceived screening norms (CSBSNorms-3). The reliabilities of these sub-scales were satisfactory with Cronbach alphas of 0.818 (CITC range, 0.652 to 0.689 ) for CSBS-Pros, 0.649 (CITC range, 0.343 to 0.497 ) for CSBS-Cons, and 0.722 (CITC range, 0.411 to 0.648 ) for CSBS-Norms.

\section{Annual exam (1-item)}

Annual exam, used as a proxy for regular cancer screenings $[14,15]$, asked a yes/no question, “Do you normally have annual health exams?"

\section{Statistical Analysis}

Chi-square tests were used to compare demographics, and $t$-tests were used to compare cancer knowledge and belief scale scores between Chinese living in the USA versus Taiwan. Logistic regressions were further used to exam how cancer education knowledge, screening beliefs, along with these key demographic factors predicted regular annual health exams.

All statistical analyses were performed using SPSS version 25.0 (IBM Corp., Armonk, NY, USA).

\section{RESULTS}

\section{Demographics}

A total of 372 Chinese adults from nine churches participated, 50\% lived in the USA and 50\% lived in Taiwan. Mean age was 44.31 (standard deviation, 14.74), with over $62 \%$ aged $40+$ years, $60 \%$ were males, $72 \%$ were married, over half reported cancer history in their families.

Current data showed that USA participants reported higher annual exam utilization (65.4\%) compared with Taiwan participants $(48.9 \%)(p=0.002)$. On the other hand, Taiwan participants were more likely to have been a cancer caregiver $(22.9 \%$ vs. $10.4 \% ; p=0.001)$ and reported doctor recommended cancer screenings ( $42.1 \%$ vs. $29.6 \% ; p=0.014$ ) (Table 1 ).

\section{Cancer Knowledge and Beliefs Among Chinese Participants in the USA vs. Taiwan}

Results showed Taiwan participants scored higher on both objective cancer education knowledge scales: CSKT (mean of 6.13 vs. $5.52 ; p<0.001$ ) and CWST (mean of 6.80 vs. $5.38 ; p<$ $0.001)$. In addition, Taiwan participants also rated higher on perceived (subjective) cancer knowledge (3.09 vs. 2.59; $p<$ 0.001). Although perceived CSBS-Pros-3 and CSBS-Cons-4 were similar, Taiwan participants endorsed higher on CSBS-Norms-3 (mean of 11.67 vs. $10.82 ; p<0.001$ ). Overall, in either group, about half of the participants (54\%) indicated had someone in their family with cancer(s), Taiwan participants however perceived higher cancer risk ( 2.77 vs. $2.56 ; p=0.013)$ and lower health status ( 3.15 vs. $3.41 ; p=0.010$ ) (Table 2 ).

Table 1. Demographics among Chinese living in the USA vs. TW

\begin{tabular}{|c|c|c|c|}
\hline Demographics & $\begin{array}{c}\text { Chinese } \\
\text { living in the } \\
\text { USA ( } n=184)\end{array}$ & $\begin{array}{c}\text { Chinese } \\
\text { living in TW } \\
(\mathbf{n}=180)\end{array}$ & $p$-value \\
\hline $\operatorname{Age}(\geq 40$ y) & 61.9 & 62.7 & NS \\
\hline Marital status (married) & 75.5 & 68.7 & NS \\
\hline Education ( $\geq$ college) & 94.5 & 75.3 & $<0.001$ \\
\hline Family cancer history (yes) & 54.3 & 53.9 & NS \\
\hline Cancer caregiver (yes) & 10.4 & 22.9 & 0.001 \\
\hline $\begin{array}{l}\text { Doctor recommend screenings } \\
\text { (yes) }\end{array}$ & 29.6 & 42.1 & 0.014 \\
\hline $\begin{array}{l}\text { Talk to doctor about screenings } \\
\text { (yes) }\end{array}$ & 23.5 & 31.5 & NS \\
\hline Annual exam (yes) & 65.4 & 48.9 & 0.002 \\
\hline
\end{tabular}


Table 2. Cancer knowledge and beliefs among Chinese living in the USA vs. TW

\begin{tabular}{lrrc}
\hline & $\begin{array}{c}\text { Chinese living } \\
\text { in the USA }\end{array}$ & $\begin{array}{c}\text { Chinese living } \\
\text { in TW }\end{array}$ & p-value \\
\hline CSKT-8 & $5.52 \pm 1.76$ & $6.13 \pm 1.21$ & $<0.001$ \\
CWST-14 & $5.38 \pm 3.26$ & $6.80 \pm 2.96$ & $<0.001$ \\
CSBS-Pros-3 items & $12.33 \pm 2.00$ & $12.71 \pm 2.20$ & NS \\
CSBS-Cons-4 items & $10.80 \pm 2.86$ & $10.71 \pm 3.72$ & $\mathrm{NS}$ \\
CSBS-Norms-3 items & $10.82 \pm 1.96$ & $11.67 \pm 2.05$ & $<0.001$ \\
Perceived health & $3.41 \pm 0.90$ & $3.15 \pm 0.91$ & 0.010 \\
Perceived cancer risk & $2.56 \pm 0.80$ & $2.77 \pm 0.79$ & 0.013 \\
Subjective cancer knowledge & $2.59 \pm 0.91$ & $3.09 \pm 0.90$ & $<0.001$ \\
\hline
\end{tabular}

Values are presented as mean \pm standard deviation.

TW, Taiwan; CSKT, Cancer Screening Knowledge Test; CWST, Cancer Warning Sign Test; CSBS, Cancer Screening Belief Scale; CSBS-Pros, CSBS-perceived screening benefits; CSBS-Cons, CSBS-perceived screening barriers; CSBSNorms, CSBS-perceived screening norms; NS, not significant.

\section{Cancer Knowledge, Beliefs, Residence Country,} and Demographics on Annual Health Exams

To exam the influence of cancer knowledge (objective and subjective), beliefs, and Chinese residence country (USA vs. Taiwan), on annual health exam (proxy for regular cancer screenings), adjusting for demographics (age, marital, education, family cancer history, and doctor recommends screenings), regression analyses was conducted. Analyses showed that Chinese participants resided in the USA were 2.38 times ( $95 \%$ confidence interval [Cl], 1.31 to 4.34) more likely to report annual health exams ( $p=0.004)$. Married participants (odd ratio $[O R], 2.85$ ), those with college education (OR, 2.38), and those indicated doctor recommending screenings $(\mathrm{OR}, 2.87)$ were also more likely to report annual health exams. Results also showed that higher CSBS-Cons-4 hindered annual health exam (OR, 0.88) (Table 3). The interaction term between CSBS-Cons-4 and country was further checked in the logistic regression model, and data showed that there was no significant interaction exist between CSBS-Cons-4 in different countries (data not shown).

\section{DISCUSSION}

Current findings show Chinese participants resided in the USA were 2.38 times more likely to report annual health exams. Married status (OR, 2.85), college education (OR, 2.38), doctor's recommendation $(\mathrm{OR}, 2.87)$, no family cancer history $(\mathrm{OR}, 2.47)$, and those with lower screening barriers were significant more likely to report annual health exam utilization. Study finding is consistent with existing literature that married
Table 3. Cancer related knowledge and beliefs, Chinese resident country, and key demographic factors on annual health exam utilization $(n=372)$

\begin{tabular}{lcccc}
\hline & B & SE & p-value & OR $(95 \%$ CI) \\
\hline Age (ref: <40 y) & 0.123 & 0.349 & NS & $1.13(0.57,2.24)$ \\
Marital (ref: single) & 1.048 & 0.359 & 0.003 & $2.85(1.41,5.76)$ \\
Education (ref: $\leq$ HS) & 0.868 & 0.418 & 0.038 & $2.38(1.05,5.41)$ \\
Cancer family history (ref: yes) & 0.905 & 0.288 & 0.002 & $2.47(1.41,4.34)$ \\
Doctor recommend screening & 1.056 & 0.316 & 0.001 & $2.87(1.55,5.34)$ \\
$\quad$ (ref: no) & & & & \\
USA-TW & 0.868 & 0.306 & 0.004 & $2.38(1.31,4.34)$ \\
Subjective knowledge & 0.210 & 0.175 & NS & $1.23(0.87,1.74)$ \\
CSKT-8 & 0.069 & 0.096 & NS & $1.07(0.89,1.29)$ \\
CWST-14 & 0.095 & 0.050 & NS & $0.91(0.82,1.00)$ \\
CSBS-Pros-3 & 0.090 & 0.075 & NS & $1.09(0.94,1.27)$ \\
CSBS-Cons-4 & -0.132 & 0.054 & 0.013 & $0.88(0.79,0.97)$ \\
CSBS-Norms-3 & 0.113 & 0.081 & NS & $1.12(0.96,1.31)$ \\
\hline
\end{tabular}

$\mathrm{SE}$, standard error; $\mathrm{OR}$, odds ratio; $\mathrm{Cl}$, confidence interval; $\mathrm{HS}$, high school; TW, Taiwan; ref: reference category; CSKT, Cancer Screening Knowledge Test; CWST, Cancer Warning Sign Test; CSBS, Cancer Screening Belief Scale; CSBS-Pros, CSBS-perceived screening benefits; CSBS-Cons, CSBS-perceived screening barriers; CSBS-Norms, CSBS-perceived screening norms; NS, not significant.

${ }^{1}$ Goodness-of-fit test $p>0.05$ indicated the logistic regression model fitted the data well; chi-square tests was statistically significant with $p<0.001$ and percentage correct $73.8 \%$ indicated good predictions of independent variables on annual health exam utilization.

and higher education were positively related to preventive health services utilization [5], and physician's recommendation being an important reinforcing factor for cancer screening among older Chinese women [4]. Lower perceived screening barriers were significantly related to higher annual exam utilization [8-11].

The association of cancer knowledge with health exam participation appeared weaker than expected. This might be partly due to the outcome measure was a proxy of any health examination, rather than a focused question about cancer screening specifically. Among the instruments, only perceived cancer screening barriers showed a significant association. Barriers measured in the current study including rather not know if had cancers, no time, no symptoms, and concerned about risks related to screening. Many of these might be common to both cancer screening and general health exam, thus showing significant relationship with annual exam participation in the current data.

Given Taiwan's universal health coverage, it is interesting to see Chinese participants in the USA were more likely to report annual health exams. In the USA, most insurance plans cover 
yearly check-ups, thus societal norms towards routine health check-ups might be higher. Yet, due to the high healthcare cost and complex copayments and deductibles, compounded with language and transportation issues, Chinese immigrants might only visit doctors when very ill or when home-remedy do not work [18]. On the other hand, the convenient, affordable, and easy healthcare access in Taiwan might results in very different utilization patterns. Recent data show that on average, Taiwan residents visit doctors (excluding dental and traditional Chinese medicine visits) about 11-12 times a year [19], whereas the average doctors' visit in the USA is only about 4 times a year [20]. Thus, although Chinese participants in the USA might have routinely gone to annual health exams, participants in Taiwan might have different healthcare seeking behaviors of being as often as they like throughout the year. Future studies are needed to test this hypothesis and investigate other potential factors.

\section{Limitations}

This study used a convenient sample of church-going Chinese adults, study sample were mostly highly educated and consisted mostly first generation Chinese-American immigrants, thus results should not be generalized to Chinese-Americans with different characteristics and background. Despite the limitations, our study provides evidence-based data for planning tailored cancer education programs. Findings have both theoretical and practical implications as it contributes to theory-based cancer screening belief applications in the field of faith-based cancer education among Chinese adults. There is a critical need to better understand and further examine factors related cancer prevention in the rapid growing and aging Chinese living overseas versus in their native countries.

In conclusion, to our knowledge, current research is the first faith-based cancer education research among Chinese midlife adults and the first comparative study between Chinese immigrants in the USA versus those resides in their native country Taiwan. Taiwan's unique universal health care environment might play a role on the higher cancer knowledge, screening norms, doctor recommendation among Taiwan participants. Findings show significant differences on key cancer prevention related factors. Study results have implication on designing effective cancer educational programs for Chinese midlife adults. Results shed light on cultural barriers and strategies for intervention development to reach and care the underserved Chinese Americans.

\section{CONFLICT OF INTEREST}

The authors have no conflicts of interest associated with the material presented in this paper.

\section{ACKNOWLEDGEMENTS}

The authors thank Mrs. Ling Jie Liu, a college alumni of the Department of Health Promotion and Behavior at the University of Georgia, for her help with data collection and data entry of this study project.

\section{ORCID}

Su-I Hou http://orcid.org/0000-0002-4519-0974

Xian Cao https://orcid.org/0000-0002-2293-7246

\section{REFERENCES}

1. Chiang CJ, Lo WC, Yang YW, You SL, Chen CJ, Lai MS. Incidence and survival of adult cancer patients in Taiwan, 2002-2012. J Formos Med Assoc 2016;115(12):1076-1088.

2. International Agency or Research on Cancer. World cancer factsheet [cited 2017 Apr 4]. Available from: https://www.cancerresearchuk.org/sites/default/files/cs_report_world.pdf.

3. Rew Research Center. The rise of Asian Americans; 2012 [cited 2017 Apr 4]. Available from: http://www.pewsocialtrends.org/ 2012/06/19/the-rise-of-asian-americans/.

4. American Cancer Society. Report: wide variation in cancer rates in Asian American/Native Hawaiian/Pacific Islanders; 2016 [cited 2017 Apr 4]. Available from: http://pressroom.cancer.org/ AANHPICancerStats2016.

5. Sentell TL, Tsoh JY, Davis T, Davis J, Braun KL. Low health literacy and cancer screening among Chinese Americans in California: a cross-sectional analysis. BMJ Open 2015;5(1):e006104.

6. Hou S. Understand barriers and explore strategies to encourage preventive health service utilization among Chinese older adults. J Georgia Public Health Assoc 2010;5(1):24-33.

7. Wen CP, Tsai SP, Chung WS. A 10-year experience with universal health insurance in Taiwan: measuring changes in health and health disparity. Ann Intern Med 2008;148(4):258-267.

8. Hou S, Hou PH, Hou HS. The impact of a colorectal cancers worksite screening intervention on knowledge, screening beliefs, and uptakes among middle-and older-age employees in Taiwan. J Cancer Educ 2014;29(Suppl 1):S12-S13. 
9. Hou SI, Fernandez ME, Baumler E, Parcel GS, Chen PH. Correlates of cervical cancer screening among women in Taiwan. Health Care Women Int 2003;24(5):384-398.

10. Hou SI, Chen PH. Home-administered fecal occult blood test for colorectal cancer screening among worksites in Taiwan. Prev Med 2004;38(1):78-84.

11. Hou SI, Fernandez ME, Baumler E, Parcel GS. Effectiveness of an intervention to increase Pap test screening among Chinese women in Taiwan. J Community Health 2002;27(4):277-290.

12. Holt CL, Wynn TA, Litaker MS, Southward P, Jeames S, Schulz E. A comparison of a spiritually based and non-spiritually based educational intervention for informed decision making for prostate cancer screening among church-attending AfricanAmerican men. Urol Nurs 2009;29(4):249-258.

13. Schwingel A, Gálvez P. Divine interventions: faith-based approaches to health promotion programs for Latinos. J Relig Health 2016;55(6):1891-1906.

14. Hou SI, Liu LJ. Objective and subjective cancer knowledge among faith-based Chinese adults. Int Q Community Health Educ 2017;38(1):47-56.

15. Hou SI. Subjective and objective cancer screening knowledge among white-and blue-collar Chinese midlife adults. J Cancer Educ 2018;33(1):167-173.

16. Hou SI. Cancer screening belief scale-Chinese version (CSBSC): validation on scale psychometric properties among a Chinese worksite population. Calif J Health Promot 2007;5(2):7999.

17. World Health Organization, Regional Office for Africa. 7 Warning signs of cancer; 2015 [cited 2017 Apr 4]. Available from: https://www.afro.who.int/news/7-warning-signs-cancer.

18. Aroian KJ, Wu B, Tran TV. Health care and social service use among Chinese immigrant elders. Res Nurs Health 2005;28(2): 95-105.

19. Cheng TM. Reflections on the 20th anniversary of Taiwan's single-payer National Health Insurance System. Health Aff (Millwood) 2015;34(3):502-510.

20. McCarthy N. Americans visit their doctor 4 times a year. People in Japan visit 13 times a year [infographic]; 2014 [cited 2017 Apr 4]. Available from: https://www.forbes.com/sites/niallmccarthy/2014/09/04/americans-visit-their-doctor-4-times-ayear-people-in-japan-visit-13-times-a-year-infographic/ \#5be53 4b9e347. 